\title{
Synthesis and pharmacological activities of some condensed 4-chloro-2,2-dialkyl chromene-3-carbaldehyde derivatives
}

\author{
MOHAMED IBRAHIM HEGAB ${ }^{1 *}$ \\ NABIL MAHMOUD YOUSEF ${ }^{1}$ \\ HANY FATHY NOUR ${ }^{1}$ \\ MOHEY ELLITHEY 2 \\ MAHMOUD SOLIMAN ARBID ${ }^{2}$ \\ ${ }^{1}$ Photochemistry Department \\ National Research Centre \\ Cairo, Egypt \\ ${ }^{2}$ Pharmacology Department \\ National Research Centre \\ Cairo, Egypt
}

\begin{abstract}
Some new hydrazono $\mathbf{5 a}, \mathbf{b}$, thiosemicarbazono $\mathbf{6 a - c}$, and oximo chromenes $7 \mathrm{a}-\mathrm{c}$ were prepared via the reaction of the corresponding $\beta$-chlorocarbaldehyde 3 with hydrazine, aromatic hydrazine, thiosemicarbazide and hydroxylamine hydrochloride, respectively. In addition, ether derivatives $\mathbf{8 a}-\mathbf{h}$ were prepared from the corresponding aldoximes 7a-c. The new products were tested for anti-inflammatory and ulcerogenic score activities compared to indomethacin.
\end{abstract}

Keywords: $\beta$-chlorocarbaldehyde, hydrazones, thiosemicarbazones, oxime ethers, anti-inflammatory activity, ulcerogenic activity

Accepted: November 21, 2007

The Vilsmeier-Haack reaction of active methylene compounds led to the formation of $\beta$-halo carbaldehydes, which constitute a class of compounds that served as useful intermediates towards construction of different heterocyclic compounds (1). Accordingly, some of $\beta$-chlorocarbaldehydes were described to react with phenylhydrazine and 2-mercapto acetic acid and its ethyl ester to give the corresponding pyrazolo and thieno derivatives (2-6). Pawar and Rajput (7) reported the synthesis of 5-anilinothiazolo[5,4-d]isoxazole from 4-chloro-2-anilinothiazol-5-carbaldehyde with hydroxylamine hydrochloride. As it is known, oxime ethers have found many uses in recent years as nonsteroidal anti-inflammatory drugs (8), insect growth regulators (9), materials with steroidal effect (10), and as antimicrobial agents (11).

In continuation of our study on hindered $\beta$-chlorocarbaldehydes (6), it seemed to be interesting to react some hindered $\beta$-chlorocarbaldehydes with some hydrazines, thiosemicarbazide, and hydroxylamine hydrochloride and to evaluate the pharmacological activity of the new products as anti-inflammatory and anti-ulcerogenic agents.

\footnotetext{
* Correspondence, e-mail: e-mail: apmihegab65@yahoo.com
} 


\section{EXPERIMENTAL}

Melting points were determined in open glass capillaries using an Electrothermal IA 9000 SERIES digital melting point apparatus (Electrothermal, UK) and are uncorrected. Microanalyses were performed with all final compounds on an Elementar-Vario EL (Elementar-Vario EL, Germany) (Microanalytical Unit, Central Services Laboratory, National Research Centre, Cairo, Egypt). The NMR spectra were recorded on a Varian Mercury VX-300 NMR spectrometer (Varian, USA). ${ }^{1} \mathrm{H}$ NMR spectra were run at $300 \mathrm{MHz}$ in $\mathrm{CDCl}_{3}$ as solvent. Chemical shifts $\delta$ are quoted in ppm and were related to that of the solvents. Mass spectra were recorded on a Shimadzu GCMS-QP 1000EX (EI, $70 \mathrm{eV}$ ) (Shimadzu, Japan) and Hewlett-Packard (EI, 70 eV) (Hewlett-Packard, USA). IR spectra were obtained with a Brucker-Vector 22 (Bruker Rhein-Stetten, Germany) for neat samples (for liquids) or $\mathrm{KBr}$ wafers (for solids).

2,3-Dihydro-2,2-dimethylchromen-4-one (1a) (12), 2,3-dihydro spirochromen(2,1') cyclohexane-4-one (1b) (13), 2,3-dihydro-2-ethyl-2-methylchromen-4-one (1c) (12), 4-chloro-2,2-dimethyl-2H-chromene (2a) (14), 4-chloro spiro-2H-chromene(2,1')cyclohexane (2b) (15), 4-chloro-2-ehyl-2-methyl-2H-chromene (2c) (16), 4-chloro-2,2-dimethyl-2H-chromene-3-carbaldehyde (3a) (6), 4-chloro spiro- $2 H$-chromene $\left(2,1^{\prime}\right)$ cyclohexane-3-carbaldehyde (3b) (6), and 4-chloro-2-ethyl-2-methyl-2H-chromene-3-carbaldehyde (3c) (16) were prepared according to the literature.

\section{Syntheses}

Reaction of $\beta$-chlorocarbaldehyde $3 c$ with hydrazine hydrate. - Hydrazine hydrate $(0.10$ $\mathrm{mL}, 2.50 \mathrm{mmol})$ was added to a solution of $\beta$-chloro carbaldehyde (3c) $(0.60 \mathrm{~g}, 1.25$ $\mathrm{mmol})$ in $99 \%$ ethanol $(10 \mathrm{~mL})$. The reaction mixture was refluxed for $3 \mathrm{~h}$, the formed solid was filtered and crystallized from ethanol to give the corresponding aldazine $R S$ $N, N^{\prime}$-bis-[1-(4-chloro-2-ethyl-2-methyl-2H-chromen-3-yl)methylidene]-hydrazine (4).

Reaction of $\beta$-chlorocarbaldehyde $3 c$ with arylhydrazine. - Arylhydrazine $(2.5 \mathrm{mmol})$ was poured into a solution of $\beta$-chlorocarbaldehyde (3c) $(0.6 \mathrm{~g}, 2.5 \mathrm{mmol})$ in $99.7 \%$ acetic acid $(15 \mathrm{~mL})$. The reaction mixture was refluxed for $3 \mathrm{~h}$ and then evaporated under reduced pressure. The residue was treated with dichloromethane, then the formed solid was filtered off, which, identified as arylhydrazine, and the filtrate was washed with $5 \%$ aqueous $\mathrm{NaHCO}_{3}$ and water, dried over $\mathrm{Na}_{2} \mathrm{SO}_{4}$, and evaporated to dryness. The residue was triturated with EtOH (few $\mathrm{mL}$ ), the yellow solid was filtered off to give the corresponding hydrazones $5 \mathbf{a}, \mathbf{b}$.

Reaction of $\beta$-chlorocarbaldehydes $3 a-c$ with thiosemicarbazide. - A mixture of $\beta$-chlorocarbaldehyde (3a, $3 \mathbf{b}$ or $3 \mathbf{c})(2 \mathrm{mmol})$ and thiosemicarbazide $(0.18 \mathrm{~g}, 2 \mathrm{mmol})$ in $99.7 \%$ acetic acid $(15 \mathrm{~mL})$ was refluxed for $3 \mathrm{~h}$ and then evaporated under reduced pressure. The residue was dissolved in dichloromethane, and the resulting solution was washed with $5 \%$ aqueous $\mathrm{NaHCO}_{3}$ and water. The organic phase was dried over $\mathrm{Na}_{2} \mathrm{SO}_{4}$ and evaporated to dryness. The crude product was purified by column chromatography on silica gel (Merck 60, particle size $0.06-0.20 \mathrm{~mm}$ ); chloroform was used as an eluent to afford the corresponding thiosemicarbazones $6 \mathbf{a}-\mathbf{c}$. 
Reaction of $\beta$-chloro carbaldehydes $3 a-c$ with hydroxylamine hydrochloride. - Hydroxylamine hydrochloride $(0.2 \mathrm{~g}, 3 \mathrm{mmol})$ was added to a solution of $\beta$-chlorocarbaldehyde $(3 \mathbf{a}, \mathbf{3 b}$ or $\mathbf{3 c})(3 \mathrm{mmol})$ in absolute ethanol $(50 \mathrm{~mL})$ containing triethylamine $(3 \mathrm{~mL})$. The reaction mixture was refluxed for $5 \mathrm{~h}$, and then the solvent was evaporated under reduced pressure. The residue was dissolved in chloroform and washed with water 3 times. The organic layer was dried over $\mathrm{Na}_{2} \mathrm{SO}_{4}$ and evaporated under reduced pressure to afford the corresponding oximes $7 \mathbf{a}-\mathbf{c}$ as crude products. The pure oximes $7 \mathbf{a}-\mathbf{c}$ were obtained after additional purification by column chromatography on silica gel (Merck 60, particle size $0.06-0.20 \mathrm{~mm})$; petroleum ether $60-80^{\circ} \mathrm{C} /$ ether $(20: 1, V / V)$ was used as an eluent.

Reaction of 4-chloro-2,2-substituted chromeno-3-aldoximes $7 \boldsymbol{a}, \boldsymbol{b}$ with acyl chlorides or ethyl chloroacetate. - An appropriate acyl chloride (acetyl chloride, chloroacetyl chloride, benzoyl chloride) or ethyl chloroacetate $(3 \mathrm{mmol})$ was added to a solution of 4 -chloro-2,2-substituted chromeno-3-aldoxime $(7 \mathbf{a}$ or $7 \mathbf{b})(3 \mathrm{mmol})$ in dry toluene $(20 \mathrm{~mL})$ containing redistilled dry pyridine $(0.25 \mathrm{~mL}, 3 \mathrm{mmol})$. The reaction mixture was refluxed for $5 \mathrm{~h}$, then cooled and extracted with water; the toluene solution was dried over calcium chloride. The solvent was evaporated under reduced pressure to give the corresponding oxime ethers $\mathbf{8 a}-\mathbf{h}$, respectively.

\section{Pharmacology}

Newly synthesized benzopyran derivatives were dissolved in $0.5 \%$ carboxymethyl cellulose (CMC) and administered intraperitonneally (i.p.), at a dose of $5 \mathrm{mg} \mathrm{kg}^{-1}$ body mass.

Wistar albino mice 3 months, $20 \mathrm{~g}$ body mass and Sprague Dawley rats ( 3 months, $100 \mathrm{~g}$ body mass) of either sex (National Research Centre, Giza, Egypt) were housed under suitable laboratory conditions $\left(30^{\circ} \mathrm{C}, \mathrm{RH} 55 \%\right)$ throughout the investigation period. Animals were fed standard pellet chow (El-Nasr Chemical Company, Cairo, Egypt) and allowed free access to water.

All animal procedures were performed after approval from the Ethics Committee of the National Research Centre and in accordance with the recommendations for the proper care and use of laboratory animals (NIH publication No. 85-23, revised 1985).

Anti-inflammatory activity. One hundred and eight rats were divided into eighteen groups, each group consisting of six animals. Anti-inflammatory activity of the compounds under investigation was studied in rats using carrageenean. A suspension of the tested compound and of the reference drug indomethacin in aqueous CMC solution $(0.5 \%, m / V)$ was administrated orally to rats at a dose level $5 \mathrm{mg} \mathrm{kg}^{-1} \mathrm{~b}$. m. Control animals were treated with $0.5 \% \mathrm{CMC}$ only. After $30 \mathrm{~min}, 0.1 \mathrm{~mL}$ of freshly prepared $1.0 \%$ carrageenean solution (in sterile $0.9 \% \mathrm{NaCl}$ ) was injected into the subplantar region of the right hind paw according to the method of Hernands-Perez et al. (17). The right paw volume was measured using a digital plethysmometer 7150 (Ugo Basile, Italy) directly before and after 1, 2, $3 \mathrm{~h}$ intervals after administration of the tested compounds. 


\section{Ulcerogenic activity}

Seventy-two rats were divided into twelve groups, each group consisting of six animals according to reported procedure (18). Ulcerogenic activity was evaluated after p.o. administration of the tested compounds or indomethacin at doses of 10,50, and $100 \mathrm{mg}$ $\mathrm{kg}^{-1}$ b. m. Control rats received vehicle per os $(0.5 \% \mathrm{CMC})$. Food but not water was removed $24 \mathrm{~h}$ before administration of the tested compounds. After $6 \mathrm{~h}$, the rats were sacrified, the stomach was removed and opened along the freater curvature, washed with distilled water and cleaned gently by dipping in saline. The mucosa damage for each stomach was examined using a stereoscopic microscope (Nikon SMZ 1B stereoscopic microscope, Nikon USA) and compared with indomethacin.

\section{Acute toxicity}

The median lethal doses $\left(L D_{50}\right)$ of the most active compounds 6 a-c were determined in mice (19). Groups of male adult mice, each of six animals, were injected i.p. with graded doses of each of the test compounds. The percentage of mortality in each group of animals was determined $24 \mathrm{~h}$ after injection. Computation of $L D_{50}$ was processed by a graphical method.

\section{RESULTS AND DISCUSSION}

\section{Chemistry}

The chromanone derivatives 1a-c were reacted with Vilsmeier reagent (DMF/ $\mathrm{POCl}_{3}$ ) to give the corresponding 4-chlorochromene derivatives $2 \mathrm{a}-\mathrm{c}$ along with 4-chloro (2H)chromene-3-carbaldehyde 3a-c according to Scheme 1.

When RS-4-chloro-2-ethyl-2-methyl $(2 H)$ chromene-3-carbaldehyde (3c) was treated with hydrazine hydrate in equimolar ratio 1:1 or in excess of hydrazine hydrate under reflux in ethanol, only the corresponding aldazine derivative 4 (Scheme 2) was separated and characterized by elemental analysis and spectral data (Tables I and II).

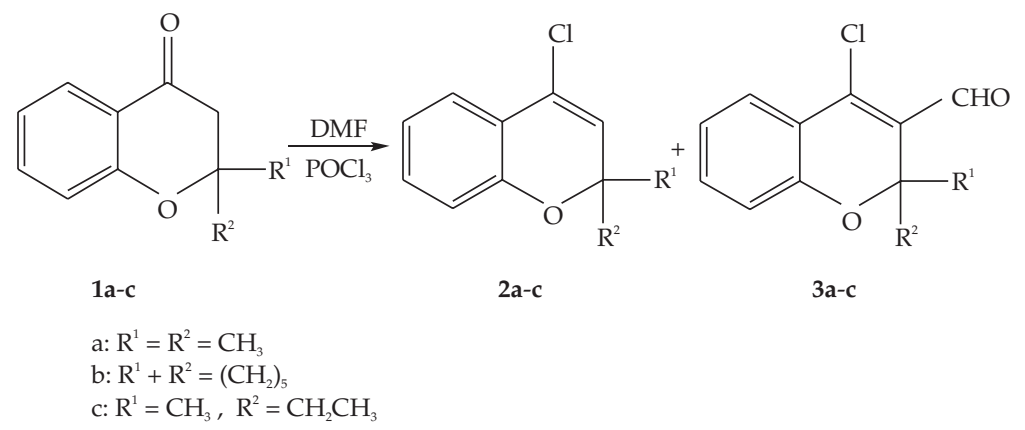


M. I. Hegab et al:: Synthesis and pharmacological activities of some condensed 4-chloro-2,2-dialkyl chromene-3-carbaldehyde derivatives, Acta Pharm. 58 (2008) 15-27.

Table I. Physical data and elemental analyses for new compounds

\begin{tabular}{|c|c|c|c|c|c|c|c|}
\hline \multirow{2}{*}{$\begin{array}{l}\text { Compd. } \\
\text { No. }\end{array}$} & \multirow{2}{*}{$\begin{array}{l}\text { Solvent for crys- } \\
\text { tallization or } \\
\text { chromatography }\end{array}$} & \multirow{2}{*}{$\begin{array}{c}\text { Physical state/ } \\
\text { m.p. }\left({ }^{\circ} \mathrm{C}\right)\end{array}$} & \multirow{2}{*}{$\begin{array}{l}\text { Yield } \\
(\%)\end{array}$} & \multirow{2}{*}{$\begin{array}{l}\text { Mol. formula } \\
\qquad\left(M_{\mathrm{r}}\right)\end{array}$} & \multicolumn{3}{|c|}{ Found/calcd. (\%) } \\
\hline & & & & & C & $\mathrm{H}$ & $\mathrm{N}$ \\
\hline \multirow[t]{2}{*}{4} & \multirow[t]{2}{*}{$\mathrm{EtOH}$} & \multirow{2}{*}{$\begin{array}{l}\text { Red crystals/ } \\
177-178\end{array}$} & \multirow[t]{2}{*}{30} & \multirow{2}{*}{$\begin{array}{c}\mathrm{C}_{26} \mathrm{H}_{26} \mathrm{Cl}_{2} \mathrm{~N}_{2} \mathrm{O}_{2} \\
(469.39)\end{array}$} & 66.21 & 5.34 & 5.79 \\
\hline & & & & & 66.52 & 5.58 & 5.96 \\
\hline \multirow[t]{2}{*}{$5 a$} & \multirow[t]{2}{*}{ PE } & \multirow{2}{*}{$\begin{array}{l}\text { Red crystals/ } \\
157-158\end{array}$} & \multirow[t]{2}{*}{5} & \multirow{2}{*}{$\begin{array}{c}\mathrm{C}_{19} \mathrm{H}_{18} \mathrm{ClN}_{3} \mathrm{O}_{3} \\
(371.80)\end{array}$} & 61.21 & 4.75 & 11.12 \\
\hline & & & & & 61.37 & 4.84 & 11.30 \\
\hline \multirow[t]{2}{*}{$5 b$} & \multirow[t]{2}{*}{$\mathrm{EtOH}$} & \multirow{2}{*}{$\begin{array}{l}\text { Red crystals/ } \\
182-183\end{array}$} & \multirow[t]{2}{*}{7} & \multirow{2}{*}{$\begin{array}{c}\mathrm{C}_{19} \mathrm{H}_{17} \mathrm{ClN}_{4} \mathrm{O}_{5} \\
(416.81)\end{array}$} & 54.53 & 3.86 & 13.09 \\
\hline & & & & & 54.74 & 4.07 & 13.44 \\
\hline \multirow[t]{2}{*}{$6 a$} & \multirow{2}{*}{$\begin{array}{l}\text { Column } \\
\left(\mathrm{CHCl}_{3}\right)\end{array}$} & \multirow{2}{*}{$\begin{array}{l}\text { Yellow crystals / } \\
249-250\end{array}$} & \multirow[t]{2}{*}{50} & $\mathrm{C}_{13} \mathrm{H}_{14} \mathrm{ClN}_{3} \mathrm{OS}$ & 52.68 & 4.70 & 14.07 \\
\hline & & & & $(295.77)$ & 52.78 & 4.73 & 14.20 \\
\hline \multirow[t]{2}{*}{$6 b$} & \multirow[t]{2}{*}{$\mathrm{MeOH}$} & Yellow crystals/ & 60 & $\mathrm{C}_{16} \mathrm{H}_{18} \mathrm{ClN}_{3} \mathrm{OS}$ & 57.31 & 5.21 & 12.35 \\
\hline & & $256-257$ & & $(335.83)$ & 57.21 & 5.35 & 12.51 \\
\hline $6 c$ & Column & Yellow crystals/ & 46 & $\mathrm{C}_{14} \mathrm{H}_{16} \mathrm{ClN}_{3} \mathrm{OS}$ & 54.18 & 5.09 & 13.41 \\
\hline & $\left(\mathrm{CHCl}_{3}\right)$ & $224-225$ & & $(309.80)$ & 54.27 & 5.16 & 13.56 \\
\hline $7 a$ & Column (PE, & Pale green & 89 & $\mathrm{C}_{12} \mathrm{H}_{12} \mathrm{ClNO}_{2}$ & 60.32 & 4.85 & 5.59 \\
\hline & ether, $20 / 3, V / V)$ & crystals/97-98 & & $(237.67)$ & 60.63 & 5.04 & 5.89 \\
\hline $7 \mathrm{~b}$ & Column (PE, & Pale green & 92 & $\mathrm{C}_{15} \mathrm{H}_{16} \mathrm{ClNO}_{2}$ & 64.73 & 5.71 & 4.86 \\
\hline & ether, $20 / 3, V / V)$ & crystals/187-188 & & $(277.73)$ & 64.86 & 5.76 & 5.04 \\
\hline $7 \mathrm{c}$ & Column (PE, & Yellow oil & 56 & $\mathrm{C}_{13} \mathrm{H}_{14} \mathrm{ClNO}_{2}$ & 61.80 & 5.41 & 5.38 \\
\hline & ether, $20 / 1, V / V)$ & & & $(25160)-$ & 62.03 & 5.56 & 5.56 \\
\hline $8 a$ & Column (PE) & Colorless oil & 52 & $\mathrm{C}_{14} \mathrm{H}_{14} \mathrm{ClNO}_{3}$ & 59.75 & 4.84 & 4.75 \\
\hline & & & & $(279.72)$ & 60.11 & 5.00 & 5.00 \\
\hline $8 b$ & $\mathrm{PE}$ & Colorless & 60 & $\mathrm{C}_{17} \mathrm{H}_{18} \mathrm{ClNO}_{3}$ & 63.53 & 5.47 & 4.05 \\
\hline & & crystals/101-103 & & $(319.78)$ & 63.84 & 5.62 & 4.37 \\
\hline $8 c$ & Column (PE) & Colorless oil & 48 & $\mathrm{C}_{14} \mathrm{H}_{13} \mathrm{Cl}_{2} \mathrm{NO}_{3}$ & 53.32 & 3.97 & 4.27 \\
\hline & & & & $(314.16)$ & 53.52 & 4.13 & 4.45 \\
\hline $8 d$ & $\mathrm{PE}$ & Colorless & 55 & $\mathrm{C}_{17} \mathrm{H}_{17} \mathrm{Cl}_{2} \mathrm{NO}_{3}$ & 57.38 & 4.49 & 3.73 \\
\hline & & crystals/106-109 & & $(354.23)$ & 57.63 & 4.79 & 3.95 \\
\hline $8 \mathrm{e}$ & Column (PE) & Colorless oil & 68 & $\mathrm{C}_{19} \mathrm{H}_{16} \mathrm{ClNO}_{3}$ & 66.42 & 4.47 & 3.86 \\
\hline & & & & $(341.79)$ & 66.76 & 4.68 & 4.09 \\
\hline $8 f$ & PE & Colorless & 80 & $\mathrm{C}_{22} \mathrm{H}_{20} \mathrm{ClNO}_{3}$ & 69.00 & 5.15 & 3.49 \\
\hline & & & & $(381.85)$ & 69.19 & 5.23 & 3.66 \\
\hline $8 \mathrm{~g}$ & Column (PE) & Colorless oil & 38 & $\mathrm{C}_{16} \mathrm{H}_{18} \mathrm{ClNO}_{4}$ & 59.05 & 5.34 & 4.11 \\
\hline & & & & $(323.77)$ & 59.35 & 5.55 & 4.32 \\
\hline $8 \mathrm{~h}$ & PE & Colorless & 50 & $\mathrm{C}_{19} \mathrm{H}_{22} \mathrm{ClNO}_{4}$ & 62.80 & 5.92 & 3.61 \\
\hline & & crystals/97-100 & & $(363.84)$ & 62.71 & 6.04 & 3.84 \\
\hline
\end{tabular}

PE - Petroleum ether $\left(40-60{ }^{\circ} \mathrm{C}\right)$ 
M. I. Hegab et al.: Synthesis and pharmacological activities of some condensed 4-chloro-2,2-dialkyl chromene-3-carbaldehyde derivatives, Acta Pharm. 58 (2008) 15-27.

Table II. Infrared, ${ }^{1} \mathrm{H}$ NMR and mass spectra

\begin{tabular}{|c|c|c|c|}
\hline $\begin{array}{l}\text { Compd. } \\
\text { No. }\end{array}$ & $\begin{array}{c}\mathrm{IR} \\
\left(v, \mathrm{~cm}^{-1}\right)\end{array}$ & $\begin{array}{l}{ }^{1} \mathrm{H} \text { NMR } \\
(\delta, \text { ppm })\end{array}$ & $\begin{array}{c}\text { MS } \\
(m / z, \%)\end{array}$ \\
\hline 4 & $\begin{array}{l}2959,2916, \\
2360,1591 \\
1479,1450 \\
1256,753\end{array}$ & $\begin{array}{l}0.95\left(\mathrm{t}, J=7.4 \mathrm{~Hz}, 6 \mathrm{H}, 2 \mathrm{CH}_{3} \mathrm{CH}_{2}\right), 1.74(\mathrm{~s}, 6 \mathrm{H}, \\
\left.2 \mathrm{CH}_{3}\right), 1.89-1.92\left(\mathrm{~m}, 2 \mathrm{H}, 2 \mathrm{CH}_{3} \mathrm{CH}_{a} \mathrm{H}_{\mathrm{b}}\right), 2.24-2.56 \\
\left(\mathrm{~m}, 2 \mathrm{H}, 2 \mathrm{CH}_{3} \mathrm{CH}_{a} \mathrm{H}_{\mathrm{b}}\right), 6.85(\mathrm{~d}, J=8.2 \mathrm{~Hz}, 2 \mathrm{H}, \\
2 \mathrm{ArH}), 6.97-7.04(\mathrm{~m}, 2 \mathrm{H}, 2 \mathrm{ArH}), 7.63-7.64(\mathrm{~m}, \\
2 \mathrm{H}, 2 \mathrm{ArH}), 7.65(\mathrm{~d}, J=7.8 \mathrm{~Hz}, 2 \mathrm{H}, 2 \mathrm{ArH}), 8.82 \\
(\mathrm{~s}, 2 \mathrm{H}, \mathrm{CH}=\mathrm{N})\end{array}$ & $\begin{array}{l}468\left(\mathrm{M}^{+},-1.5\right), 433 \\
(56), 403(16), 397 \\
(24), 255(60), 221 \\
(33), 219(100), 204 \\
(33), 183(16), 178 \\
(27)\end{array}$ \\
\hline $5 a$ & $\begin{array}{l}3450,1598, \\
1478,1312, \\
1267,1106, \\
748\end{array}$ & $\begin{array}{l}0.90\left(\mathrm{t}, J=8.1 \mathrm{~Hz}, 3 \mathrm{H}, \mathrm{CH}_{3} \mathrm{CH}_{2}\right), 1.68\left(\mathrm{~s}, 3 \mathrm{H}, \mathrm{CH}_{3}\right), \\
1.80-1.93\left(\mathrm{~m}, 2 \mathrm{H}, \mathrm{CH}_{3} \mathrm{CH}_{2}\right), 6.86(\mathrm{~d}, J=7.9 \mathrm{~Hz}, \\
1 \mathrm{H}, \mathrm{ArH}), 6.98-7.07(\mathrm{~m}, 3 \mathrm{H}, \mathrm{ArH}), 7.27-7.32 \\
(\mathrm{~m}, 1 \mathrm{H}, \mathrm{ArH}), 7.49(\mathrm{~d}, J=6.9 \mathrm{~Hz}, 1 \mathrm{H}, \mathrm{ArH}), \\
8.15(\mathrm{~d}, J=9.07 \mathrm{~Hz}, 2 \mathrm{H}, \text { ArH }), 8.27(\mathrm{~s}, 1 \mathrm{H}, \mathrm{CH}=\mathrm{N}), \\
11.52\left(\mathrm{~s}, 1 \mathrm{H}, \mathrm{NH}, \text { exchangeable with } \mathrm{D}_{2} \mathrm{O}\right)\end{array}$ & $\begin{array}{l}373\left(\mathrm{M}^{+} \mathrm{Cl}^{37}, 6\right), 371 \\
\left(\mathrm{M}^{+} \mathrm{Cl}^{35}, 22\right), 342 \\
(54), 306(100), 260 \\
(24), 204(23), 178 \\
(14)\end{array}$ \\
\hline $5 b$ & $\begin{array}{l}3267,2940 \\
1596,1501 \\
134,1136 \\
1091,760\end{array}$ & $\begin{array}{l}0.90\left(\mathrm{t}, J=8.1 \mathrm{~Hz}, 3 \mathrm{H}, \mathrm{CH}_{3} \mathrm{CH}_{2}\right), 1.68\left(\mathrm{~s}, 3 \mathrm{H}, \mathrm{CH}_{3}\right), \\
1.83-1.91\left(\mathrm{~m}, 2 \mathrm{H}, \mathrm{CH}_{3} \mathrm{CH}_{2}\right), 6.89(\mathrm{~d}, J=8.1 \mathrm{~Hz}, \\
1 \mathrm{H}, \mathrm{ArH}), 7.03(\mathrm{t}, \mathrm{J}=7.6 \mathrm{~Hz}, 1 \mathrm{H}, \mathrm{ArH}), 7.32-7.37 \\
(\mathrm{~m}, 1 \mathrm{H}, \mathrm{ArH}), 7.54(\mathrm{~d}, J=6.9 \mathrm{~Hz}, 1 \mathrm{H}, \mathrm{ArH}), 7.77 \\
(\mathrm{~d}, J=9.5 \mathrm{~Hz}, 1 \mathrm{H}, \mathrm{ArH}), 8.43(\mathrm{dd}, \mathrm{J}=9.5,2.5 \mathrm{~Hz}, \\
1 \mathrm{H}, \mathrm{ArH}), 8.85(\mathrm{~s}, 1 \mathrm{H}, \mathrm{ArH}), 8.96(\mathrm{~s}, 1 \mathrm{H}, \mathrm{CH}=\mathrm{N}), \\
11.85\left(\mathrm{~s}, 1 \mathrm{H}, \mathrm{NH}, \text { exchangeable with } \mathrm{D}_{2} \mathrm{O}\right)\end{array}$ & $\begin{array}{l}418\left(\mathrm{M}^{+} \mathrm{Cl}^{37}, 9\right), 416 \\
\left(\mathrm{M}^{+} \mathrm{Cl}^{35}, 27\right), 387 \\
(100), 351(97), 204 \\
(44), 178(22), 170 \\
(23)\end{array}$ \\
\hline $6 a$ & $\begin{array}{l}3427,3259, \\
3153,2975, \\
1593,1529, \\
1454,1366, \\
1289,1099, \\
750\end{array}$ & $\begin{array}{l}1.63\left(\mathrm{~s}, 6 \mathrm{H}, 2 \mathrm{CH}_{3}\right), 6.86(\mathrm{~d}, J=8.1 \mathrm{~Hz}, 1 \mathrm{H}, \mathrm{ArH}) \\
7.00-7.05(\mathrm{~m}, 1 \mathrm{H}, \mathrm{ArH}), 7.29-7.34(\mathrm{~m}, 1 \mathrm{H}, \mathrm{ArH}) \\
7.44\left(\mathrm{~s}, \mathrm{H}, \mathrm{NH}, \text { exchangeable with } \mathrm{D}_{2} \mathrm{O}\right), 7.50 \\
(\mathrm{~d}, J=7.8 \mathrm{~Hz}, 1 \mathrm{H}, \mathrm{ArH}), 8.29(\mathrm{~s}, \mathrm{H}, \mathrm{NH}, \\
\left.\text { exchangeable with } \mathrm{D}_{2} \mathrm{O}\right), 8.33(\mathrm{~s}, 1 \mathrm{H}, \mathrm{CH}=\mathrm{N}) \\
11.52\left(\mathrm{~s}, 1 \mathrm{H}, \mathrm{NH}, \text { exchangeable with } \mathrm{D}_{2} \mathrm{O}\right)\end{array}$ & $\begin{array}{l}297\left(\mathrm{M}^{+} \mathrm{Cl}^{37}, 6\right), 295 \\
\left(\mathrm{M}^{+} \mathrm{Cl}^{35}, 21\right), 219 \\
(16), 204(100), 185 \\
(54), 115(13), 60 \\
(31)\end{array}$ \\
\hline $6 b$ & $\begin{array}{l}3430,3270, \\
3152,2930, \\
1590,1528, \\
1448,1289, \\
1244,1113, \\
757\end{array}$ & $\begin{array}{l}1.47-1.69\left(\mathrm{~m}, 8 \mathrm{H}, 2 \mathrm{CH}_{2}\right), 1.82(\mathrm{~d}, J=12.0 \mathrm{~Hz}, \\
\left.2 \mathrm{H}, \mathrm{CH}_{2}\right), 6.95(\mathrm{~d}, J=8.1 \mathrm{~Hz}, 1 \mathrm{H}, \text { ArH }), 7.01-7.06 \\
(\mathrm{~m}, 1 \mathrm{H}, \mathrm{ArH}), 7.27(\mathrm{~s}, \mathrm{H}, \mathrm{NH}, \text { exchangeable with } \\
\left.\mathrm{D}_{2} \mathrm{O}\right), 7.31-7.34(\mathrm{~m}, 1 \mathrm{H}, \mathrm{ArH}), 7.49(\mathrm{dd}, J=7.8, \\
\text { 1.5 Hz, } 1 \mathrm{H}, \mathrm{ArH}), 8.28(\mathrm{~s}, \mathrm{H}, \mathrm{NH}, \text { exchangeable } \\
\left.\text { with } \mathrm{D}_{2} \mathrm{O}\right), 8.37(\mathrm{~s}, 1 \mathrm{H}, \mathrm{CH}=\mathrm{N}), 11.53(\mathrm{~s}, 1 \mathrm{H}, \mathrm{NH}, \\
\left.\text { exchangeable with } \mathrm{D}_{2} \mathrm{O}\right)\end{array}$ & $\begin{array}{l}337\left(\mathrm{M}^{+} \mathrm{Cl}^{37}, 8\right), 335 \\
\left(\mathrm{M}^{+} \mathrm{Cl}^{35}, 24\right), 259 \\
(53), 216(100), 203 \\
(20), 197(27), 115 \\
(15), 60(47)\end{array}$ \\
\hline $6 c$ & $\begin{array}{l}3442,3270, \\
3150,2974, \\
2362,1592, \\
1530,1454, \\
1372,1290, \\
1101,753\end{array}$ & $\begin{array}{l}0.85\left(\mathrm{t}, J=7.3 \mathrm{~Hz}, 3 \mathrm{H}, \mathrm{CH}_{3} \mathrm{CH}_{2}\right), 1.59\left(\mathrm{~s}, 3 \mathrm{H}, \mathrm{CH}_{3}\right), \\
1.74-1.83\left(\mathrm{~m}, 1 \mathrm{H}, \mathrm{CH}_{3} \mathrm{CH}_{a} \mathrm{H}_{\mathrm{b}}\right), 2.24-2.33(\mathrm{~m}, 1 \mathrm{H}, \\
\left.\mathrm{CH}_{3} \mathrm{CH}_{\mathrm{a}} \mathrm{H}_{b}\right), 6.85(\mathrm{dd}, J=8.1,1.2 \mathrm{~Hz}, 1 \mathrm{H}, \mathrm{ArH}), \\
7.00-7.04(\mathrm{~m}, 1 \mathrm{H}, \mathrm{ArH}), 7.30-7.38(\mathrm{~m}, 1 \mathrm{H}, \mathrm{ArH}), \\
7.40\left(\mathrm{~s}, \mathrm{H}, \mathrm{NH}, \text { exchangeable with } \mathrm{D}_{2} \mathrm{O}\right), 7.49 \\
(\mathrm{dd}, J=7.8,1.5 \mathrm{~Hz}, 1 \mathrm{H}, \text { ArH }), 8.28(\mathrm{~s}, 1 \mathrm{H}, \mathrm{NH}, \\
\left.\text { exchangeable with } \mathrm{D}_{2} \mathrm{O}\right), 8.37(\mathrm{~s}, 1 \mathrm{H}, \mathrm{CH}=\mathrm{N}), \\
11.51\left(\mathrm{~s}, 1 \mathrm{H}, \mathrm{NH}, \text { exchangeable with } \mathrm{D}_{2} \mathrm{O}\right)\end{array}$ & $\begin{array}{l}311\left(\mathrm{M}^{+} \mathrm{Cl}^{37}, 5\right), 309 \\
\left(\mathrm{M}^{+} \mathrm{Cl}^{35}, 17\right), 204 \\
(93), 185(100), 128 \\
(11), 115(12), 60(41)\end{array}$ \\
\hline $7 a$ & $\begin{array}{l}3282,1592, \\
1474,1296, \\
1256,975 \\
764\end{array}$ & $\begin{array}{l}1.65\left(\mathrm{~s}, 6 \mathrm{H}, 2 \mathrm{CH}_{3}\right), 6.82(\mathrm{~d}, J=8.1 \mathrm{~Hz}, 1 \mathrm{H}, \mathrm{ArH}) \\
6.93-6.98(\mathrm{~m}, 1 \mathrm{H}, \mathrm{ArH}), 7.21-7.27(\mathrm{~m}, 1 \mathrm{H}, \mathrm{ArH}) \\
7.45\left(\mathrm{~s}, \mathrm{H}, \mathrm{OH}, \text { exchangeable with } \mathrm{D}_{2} \mathrm{O}\right), 7.53 \\
(\mathrm{~d}, J=7.6 \mathrm{~Hz}, 1 \mathrm{H}, \text { ArH }), 8.39(\mathrm{~s}, 1 \mathrm{H}, \mathrm{CH}=\mathrm{N})\end{array}$ & $\begin{array}{l}239\left(\mathrm{M}^{+} \mathrm{Cl}^{37}, 7\right), 237 \\
\left(\mathrm{M}^{+} \mathrm{Cl}^{35}, 27\right), 222 \\
(100), 204(25), 186 \\
(36), 180(28), 178 \\
(16), 115(20), 77 \\
(22), 63(29)\end{array}$ \\
\hline
\end{tabular}


7b $\quad 3262,2928, \quad 1.23-2.19\left(\mathrm{~m}, 10 \mathrm{H}, 5 \mathrm{CH}_{2}\right), 6.89(\mathrm{~d}, J=7.9 \mathrm{~Hz}$, $1590,1474, \quad 1 \mathrm{H}, \mathrm{ArH}), 6.93-6.99(\mathrm{~m}, 1 \mathrm{H}, \mathrm{ArH}), 7.22-7.25$ 1445, 1288, (m, 1H, ArH), 7.44 (s, H, OH, exchangeable 1258, 978, with $\left.\mathrm{D}_{2} \mathrm{O}\right), 7.53(\mathrm{dd}, J=7.6,1.3 \mathrm{~Hz}, 1 \mathrm{H}, \mathrm{ArH})$, 757

$7 c$ $-$ $8.35(\mathrm{~s}, 1 \mathrm{H}, \mathrm{CH}=\mathrm{N})$

$0.83\left(\mathrm{t}, \mathrm{J}=7.3 \mathrm{~Hz}, 3 \mathrm{H}, \mathrm{CH}_{3} \mathrm{CH}_{2}\right), 1.50$ $\left(\mathrm{s}, 3 \mathrm{H}, \mathrm{CH}_{3}\right), 1.84-1.89\left(\mathrm{~m}, 1 \mathrm{H}, \mathrm{CH}_{3} \mathrm{CH}_{a} \mathrm{H}_{\mathrm{b}}\right)$, 2.31-2.36 (m, $\left.1 \mathrm{H}, \mathrm{CH}_{3} \mathrm{CH}_{\mathrm{a}} \mathrm{H}_{b}\right), 6.70(\mathrm{~d}, J=8.1$, $\mathrm{Hz}, 1 \mathrm{H}, \mathrm{ArH}), 6.86-6.99$ (m, 1H, ArH) , 7.23-7.58 (m, 1H, ArH), $7.41(\mathrm{~d}, J=7.9 \mathrm{~Hz}, 1 \mathrm{H}, \mathrm{ArH})$, 8.31 (s, H, OH, exchangeable with $\left.\mathrm{D}_{2} \mathrm{O}\right), 8.47$ (s, $1 \mathrm{H}, \mathrm{CH}=\mathrm{N})$

2931, 2925, 1.65 (s, 6H, $\left.2 \mathrm{CH}_{3}\right), 2.38\left(\mathrm{~s}, 3 \mathrm{H}, \mathrm{CH}_{3}\right), 6.80$ 1730, 1615, $(\mathrm{d}, J=8.1 \mathrm{~Hz}, 1 \mathrm{H}, \mathrm{ArH}), 6.91-6.96(\mathrm{~m}, 1 \mathrm{H}$, 1474, 1445, ArH), 7.21-7.27 (m, 1H, ArH), 7.51 $1288,1258, \quad(\mathrm{~d}, J=7.6 \mathrm{~Hz}, 1 \mathrm{H}, \mathrm{ArH}), 8.43$ 978,757 $(\mathrm{s}, 1 \mathrm{H}, \mathrm{CH}=\mathrm{N})$ 978,759 971, 765

1.20-2.29 (m, 10H, $\left.5 \mathrm{CH}_{2}\right), 2.39\left(\mathrm{~s}, 3 \mathrm{H}, \mathrm{CH}_{3}\right)$, $6.89(\mathrm{~d}, J=8.1 \mathrm{~Hz}, 1 \mathrm{H}, \mathrm{ArH}), 6.91-6.96$ (m, 1H, ArH), 7.21-7.27 (m, 1H, ArH), 7.51 $(\mathrm{d}, J=7.6 \mathrm{~Hz}, 1 \mathrm{H}, \mathrm{ArH}), 8.42(\mathrm{~s}, 1 \mathrm{H}, \mathrm{CH}=\mathrm{N})$ 978,757

2933, 2925, $1.20-2.29\left(\mathrm{~m}, 10 \mathrm{H}, 5 \mathrm{CH}_{2}\right), 6.89(\mathrm{~d}, J=8.1 \mathrm{~Hz}$, 1733, 1623, 1H, ArH), 6.91-6.96 (m, 1H, ArH), 7.21-7.27 $1474,1445, \quad(\mathrm{~m}, 6 \mathrm{H}, \mathrm{ArH}), 7.51(\mathrm{~d}, J=7.6 \mathrm{~Hz}, 1 \mathrm{H}, \mathrm{ArH})$, $1286,1254, \quad 8.43(\mathrm{~s}, 1 \mathrm{H}, \mathrm{CH}=\mathrm{N})$

$279\left(\mathrm{M}^{+} \mathrm{Cl}^{37}, 5\right), 277$ $\left(\mathrm{M}^{+} \mathrm{Cl}^{35}, 16\right), 260$ (100), 217 (41), 204 (35), 115 (12), 77 (13), 63 (16)

$281\left(\mathrm{M}^{+} \mathrm{Cl}^{37}, 2\right), 279$ $\left(\mathrm{M}^{+}, 8\right), 221(32)$, 219 (100), 206 (7), $204(25), 186(36)$, 180 (28), 178 (16), 115 (20), 77 (22), 63 (29)

$321\left(\mathrm{M}^{+} \mathrm{Cl}^{37}, 2\right), 319$ $\left(\mathrm{M}^{+}, 10\right), 261(33)$, 259 (100), 233 (23), 231 (80), 206 (21), 204 (65), 186 (26), 180 (27), 178 (26), 115 (25), 77 (23), 63 (30) 223 (25), 221 (100), 206 (9), 204 (30), 186 (34), 180 (26), 178 (18), 115 (22), 77 (24), 63 (27) 261 (29), 259 (100), 233 (21), 231 (78), 206 (23), 204 (65), 186 (26), 180 (27), 178 (26), 115 (25), 77 (23), 63 (30)

$343\left(\mathrm{M}^{+} \mathrm{Cl}^{37}, 3\right), 341$ $\left(\mathrm{M}^{+} \mathrm{Cl}^{35}, 12\right), 238$ (32), 240 (100), 206 (9), 204 (27), 186 (38), 180 (32), 178 (20), 115 (22), 77 (24), 63 (39)

$383\left(\mathrm{M}^{+} \mathrm{Cl}^{37}, 3\right), 381$ $\left(\mathrm{M}^{+} \mathrm{Cl}^{35}, 15\right), 278$

(33), 276 (100), 233 (23), 231 (80), 206 (21), 204 (65), 186 (28), 180 (27), 178 (26), 115 (25), 77 (27), 63 (40) 

vatives, Acta Pharm. 58 (2008) 15-27.

$\begin{array}{llll}8 \mathrm{~g} & 2935,2925, & 0.93\left(\mathrm{t}, \mathrm{J}=8.0 \mathrm{~Hz}, 3 \mathrm{H}, \mathrm{CH}_{2} \mathrm{CH}_{3}\right), & 223(15), 221(50), \\ & 2926,2889, & 1.65\left(\mathrm{~s}, 6 \mathrm{H}, 2 \mathrm{CH}_{3}\right), 4.27\left(\mathrm{~m}, 2 \mathrm{H}, \mathrm{CH}_{2} \mathrm{CH}_{3}\right), & 206(31), 204(100), \\ 1720,1615, & 4.46\left(\mathrm{~s}, 2 \mathrm{H}, \mathrm{CH}_{2}\right), 6.79(\mathrm{~d}, \mathrm{~J}=8.1 \mathrm{~Hz}, 1 \mathrm{H}, \mathrm{ArH}), & 186(34), 180(26), \\ & 1475,1439, & 6.91-6.95(\mathrm{~m}, 1 \mathrm{H}, \mathrm{ArH}), 7.22-7.27(\mathrm{~m}, 1 \mathrm{H}, \mathrm{ArH}), & 178(18), 115(24), 77 \\ 1294,1262, & 7.52(\mathrm{~d}, \mathrm{~J}=7.6 \mathrm{~Hz}, 1 \mathrm{H}, \mathrm{ArH}), & (24), 63(29) \\ & 971,765 & 8.44(\mathrm{~s}, 1 \mathrm{H}, \mathrm{CH}=\mathrm{N}) & 261(17), 259(55), \\ \mathbf{8 h} & 2925,2926, & 0.93\left(\mathrm{t}, \mathrm{J}=8.0 \mathrm{~Hz}, 3 \mathrm{H}, \mathrm{CH}_{2} \mathrm{CH}_{3}\right), 1.21-2.31 & 233(21), 231(78), \\ & 1720,1615, & (\mathrm{~m}, 10 \mathrm{H}, 5 \mathrm{CH}), 4.25\left(\mathrm{~m}, 2 \mathrm{H}, \mathrm{CH} \mathrm{CH}_{3}\right), 4.44 & 206(27), 204(100), \\ 1475,1439, & (\mathrm{~s}, 2 \mathrm{H}, \mathrm{CH}), 6.88(\mathrm{~d}, \mathrm{~J}=8.1 \mathrm{~Hz}, 1 \mathrm{H}, \mathrm{ArH}), & 186(26), 180(27), \\ 1294,1262, & 6.90-6.95(\mathrm{~m}, 1 \mathrm{H}, \mathrm{ArH}), 7.21-7.27(\mathrm{~m}, 1 \mathrm{H}, \mathrm{ArH}), & 178(26), 115(27), 77 \\ 971,765 & 7.50(\mathrm{~d}, J=7.6 \mathrm{~Hz}, 1 \mathrm{H}, \mathrm{ArH}), 8.42(\mathrm{~s}, 1 \mathrm{H}, \mathrm{CH}=\mathrm{N}) & (23), 63(32)\end{array}$

PE - Petroleum ether

The IR spectrum of compound 4 showed $\mathrm{C}=\mathrm{N}$ absorption at $1591 \mathrm{~cm}^{-1}$, whereas ${ }^{1} \mathrm{H}$ NMR spectrum of 4 showed the methylidine proton at $\delta 8.82 \mathrm{ppm}$. The mass spectrum of 4 showed a prominent ion peak $\mathrm{M}^{+}$at $m / z 468(5 \%)$.

Accordingly, RS-4-chloro-2-ethyl-2-methyl $(2 H)$ chromene-3-carboxaldehyde (3c) reacted with 4-nitrophenylhydrazine and 2,4-dinitrophenylhydrazine to produce the corresponding hydrazone derivatives $\mathbf{5 a}, \mathbf{b}$ (Scheme 2 ).

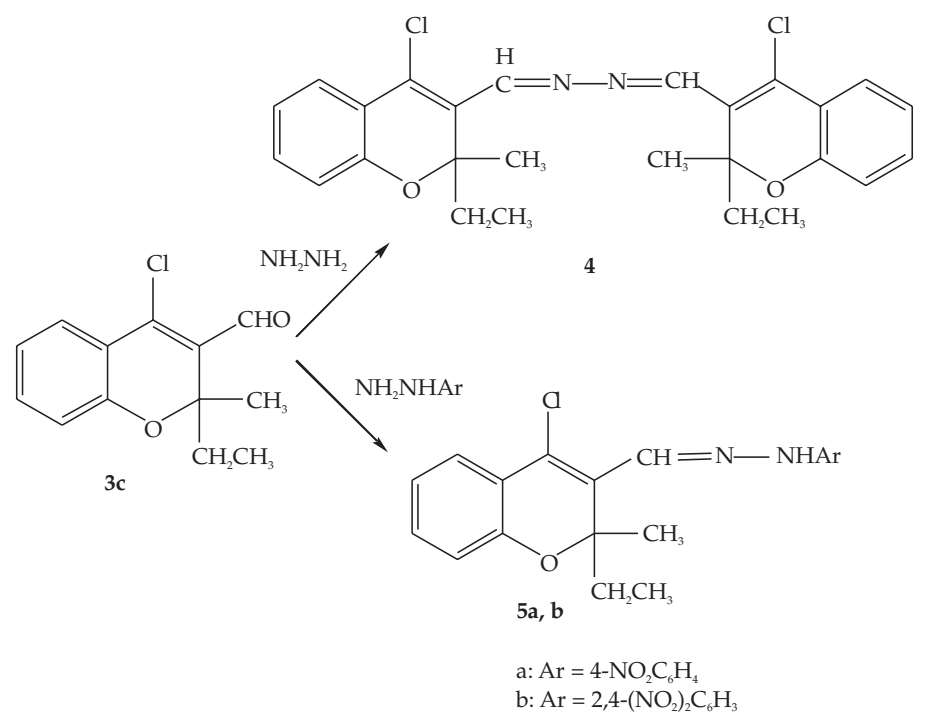

Scheme 2 
The elemental analyses and spectral data revealed the required structures. The IR spectra of $\mathbf{5 a}, \mathbf{b}$ showed $\mathrm{C}=\mathrm{N}$ at 1598 and $1596 \mathrm{~cm}^{-1}$, respectively. The ${ }^{1} \mathrm{H}$ NMR spectra of $5 \mathbf{a}, \mathbf{b}$ showed methylidine protons at $\delta 8.27,8.96$ and NH at $\delta 11.52,11.85 \mathrm{ppm}$ (exchangeable with $\mathrm{D}_{2} \mathrm{O}$ ). The mass spectra of $\mathbf{5} \mathbf{a}, \mathbf{b}$ showed prominent ion peaks $\mathrm{M}^{+}$at $\mathrm{m} / \mathrm{z} 371$ $\left(\mathrm{M}^{+} \mathrm{Cl}^{35}, 22 \%\right)$ and $416\left(\mathrm{M}^{+} \mathrm{Cl}^{35}, 27 \%\right)$, respectively.

Some of the semicarbazones and thiosemicarbazones have been reported as biologically active compounds $(20,21)$. So, 4 -chloro $(2 H)$ chromene-3-carbaldehydes 3a-c reacting with thiosemicarbazide in acetic acid under reflux for $3 \mathrm{~h}$ affored the corresponding thiosemicarbazones 6a-c (Scheme 3). The IR spectra of 6a-c show $\mathrm{NH}_{2}, \mathrm{NH}$ at 3442-3152, $\mathrm{C}=\mathrm{N}$ at $1593-1590 \mathrm{~cm}^{-1}$. The ${ }^{1} \mathrm{H}$ NMR spectra of $6 \mathrm{a}-\mathrm{c}$ showed the two protons of $\mathrm{NH}_{2}$ as magnetically un-equivalent protons, whereas they appeared at 7.27-7.44, 8.28-8.29 ppm (exchangeable with $\mathrm{D}_{2} \mathrm{O}$ ), methylidine protons at 8.33-8.37 ppm and $\mathrm{NH}$ at 11.51-11.53 ppm (exchangeable with $\mathrm{D}_{2} \mathrm{O}$ ). The mass spectra of 6a-c showed prominent ion peaks

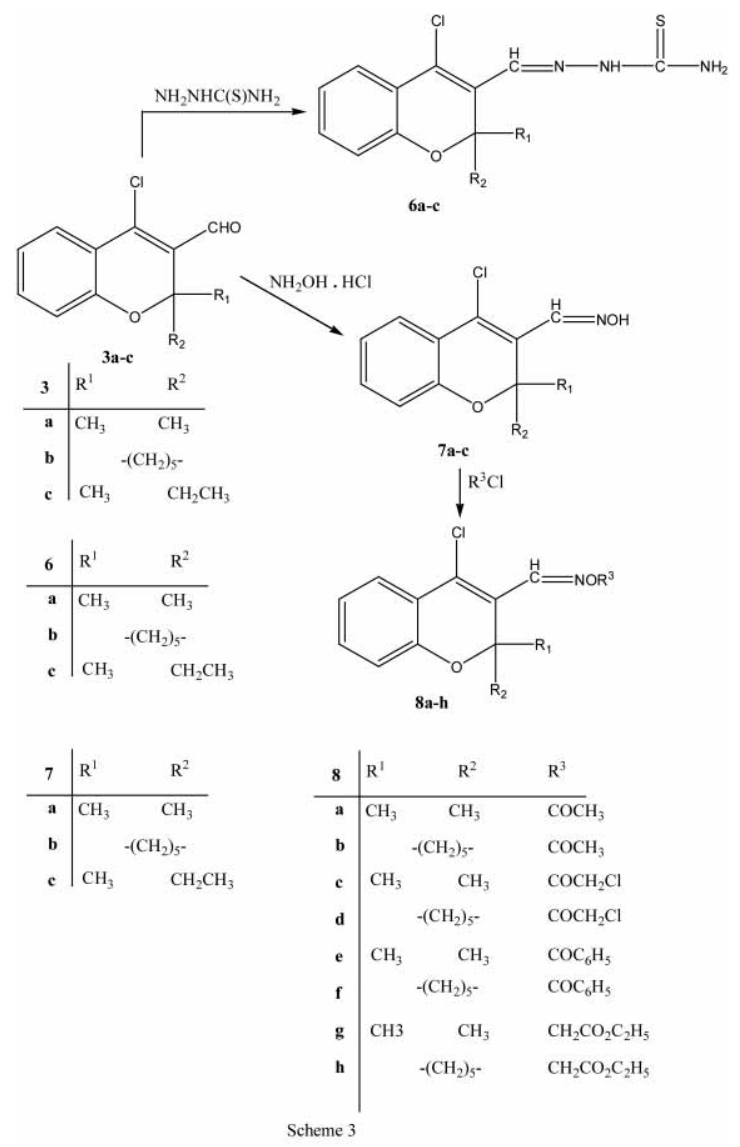

Scheme 3 
$\mathrm{M}^{+}$at $\mathrm{m} / \mathrm{z} 295\left(\mathrm{M}^{+} \mathrm{Cl}^{35}, 21 \%\right)$ and $335\left(\mathrm{M}^{+} \mathrm{Cl}^{35}, 24 \%\right), 309\left(\mathrm{M}^{+} \mathrm{Cl}^{35}, 17 \%\right)$, respectively. The thiosemicarbazones 6a-c were not changed after heating with $\mathrm{KOH}$ in ethanol under reflux for $10 \mathrm{~h}$.

Reaction of $\beta$-chlorocarbaldehydes 3a-c with hydroxylamine hydrochloride in absolute ethanol containing triethylamine under reflux for $5 \mathrm{~h}$ afforded the corresponding oxime derivatives 7a-c (Scheme 3). The IR spectra of $\mathbf{7 a}, \mathbf{b}$ show $\mathrm{OH}$ at $3282,3262, \mathrm{C}=\mathrm{N}$ at $1592,1590 \mathrm{~cm}^{-1}$. The ${ }^{1} \mathrm{H}$ NMR spectra of $7 \mathrm{a}-\mathrm{c}$ showed $\mathrm{OH}$ at 7.45, 7.44, $8.31 \mathrm{ppm}$ (exchangeable with $\mathrm{D}_{2} \mathrm{O}$ ), respectively, and methylidine protons at $8.39,8.35,8.47 \mathrm{ppm}$, respectively. The mass spectra of $7 \mathbf{a}, \mathbf{b}$ showed prominent ion peaks $\mathbf{M}^{+}$at $\mathrm{m} / z 237\left(\mathrm{M}^{+} \mathrm{Cl}^{35}\right.$, $27 \%)$ and $277\left(\mathrm{M}^{+} \mathrm{Cl}^{35}, 16 \%\right)$, respectively. However, the oximes $7 \mathbf{a}, \mathbf{b}$ reacted with some acyl chlorides, namely, acetyl chloride, chloroacetyl chloride, benzoyl chloride and ethyl chloroacetate in dry toluene in the presence of redistilled dry pyridine to give the corresponding $\mathrm{O}$-acyloxime and ethoxy carbamoyl methyl derivatives 8a-h (Scheme 3). The

Table III. Anti-inflammatory activity of the synthesized compounds

\begin{tabular}{lccc}
\hline \multirow{2}{*}{$\begin{array}{c}\text { Compd. } \\
\text { No. }\end{array}$} & \multicolumn{3}{c}{ Edema inhibition $(\text { mean } \pm \text { S.E.M })^{\mathrm{a}, \mathrm{b}}(\%)$} \\
\cline { 2 - 4 } Control & $15.5 \pm 4.3$ & $2 \mathrm{~h}$ & $3 \mathrm{~h}$ \\
$\mathbf{4}$ & $17.4 \pm 4.8$ & $14.9 \pm 4.5$ & $17.2 \pm 4.4$ \\
$\mathbf{5 a}$ & $16.1 \pm 5.6$ & $30.0 \pm 4.8$ & $36.5 \pm 3.4$ \\
$\mathbf{5 b}$ & $13.1 \pm 5.0$ & $27.8 \pm 5.8$ & $34.8 \pm 5.6$ \\
$\mathbf{6 a}$ & $39.7 \pm 1.9$ & $14.7 \pm 3.9$ & $31.4 \pm 4.9$ \\
$\mathbf{6 b}$ & $34.6 \pm 2.3$ & $52.6 \pm 1.6$ & $59.4 \pm 1.9$ \\
$\mathbf{6 c}$ & $37.7 \pm 1.4$ & $47.6 \pm 1.8$ & $54.5 \pm 1.6$ \\
$\mathbf{7 a}$ & $8.8 \pm 7.1$ & $55.6 \pm 1.6$ & $62.4 \pm 1.9$ \\
$\mathbf{7 b}$ & $15.7 \pm 5.6$ & $21.7 \pm 6.4$ & $28.4 \pm 7.1$ \\
$\mathbf{7 c}$ & $12.8 \pm 6.1$ & $28.6 \pm 5.0$ & $35.3 \pm 5.6$ \\
$\mathbf{8 a}$ & $10.8 \pm 7.1$ & $25.7 \pm 6.4$ & $32.4 \pm 5.6$ \\
$\mathbf{8 b}$ & $17.7 \pm 5.5$ & $23.8 \pm 6.4$ & $30.5 \pm 7.1$ \\
$\mathbf{8 c}$ & $15.9 \pm 2.3$ & $30.5 \pm 5.7$ & $37.3 \pm 5.5$ \\
$\mathbf{8 d}$ & $21.8 \pm 4.8$ & $28.8 \pm 2.4$ & $35.5 \pm 1.2$ \\
$\mathbf{8 e}$ & - & $34.8 \pm 4.8$ & $41.4 \pm 3.4$ \\
$\mathbf{8 f}$ & - & - & - \\
8g & - & - & - \\
8h & - & - & - \\
Indomethacin & $42.8 \pm 5.7$ & $55.7 \pm 6.3$ & - \\
\hline
\end{tabular}

(-) - No activity.

a Dose: $5 \mathrm{mg} \mathrm{kg}^{-1}$ b.m. (p.o.).

b $n=6$. 
analytical and spectroscopic characterization data of compounds 8a-h given in Tables I and II are in good agreement with the expected structural characteristics.

\section{Anti-inflammatory activity}

The results of ant-inflammatory testing are listed in Table III, showing the percent inhibition of edema obtained by the reference drug and tested compounds, respectively. Results show that compounds $\mathbf{4}, \mathbf{5 a}, \mathbf{b}, \mathbf{7 a}, \mathbf{b}, \mathbf{c}$ and $\mathbf{8 a}, \mathbf{b}, \mathbf{c}, \mathbf{d}$ possess weak anti-inflammatory activity (8-41\% of inhibition) in comparison to that of indomethacin (42-62\%). Compounds $6 \mathbf{a}, \mathbf{6 b}$ and $\mathbf{6 c}$ showed activity of 35 to $62 \%$, equal to that of indomethacin. The condensation of thiosemicarbazide group with carbaldehyde group in the pyrane ring increased the activity in all cases. Cyclohexyl group at position 2 in the pyrane ring was found to be less active compared to methyl and ethyl groups at position 2 in the pyrane ring (Table III).

\section{Ulcerogenicity and acute toxicity}

Compounds 6a-c were screened for their ulcerogenic activity at dose levels of 10, 50 and $100 \mathrm{mg} \mathrm{kg}^{-1}$ b.m. (Table IV). The Tested Compounds 6a-c Showed No Ulcerogenic Activity compared to indomethacin, which showed mean ulcerogenic activity of 1.4 to $2.1 \mathrm{~mm}$.

Table IV. Gastric ulceration in rats ${ }^{a}$

\begin{tabular}{lccc}
\hline $\begin{array}{l}\text { Compd. } \\
\text { No. }\end{array}$ & \multicolumn{3}{c}{ Dose $\left(\mathrm{mg} \mathrm{kg}^{-1}\right)$} \\
\cline { 2 - 4 } & 10 & 50 & 100 \\
\hline Control & $0 / 6$ & $0 / 6$ & $0 / 6$ \\
6a & $0 / 6(0)$ & $0 / 6(0)$ & $0 / 6(0)$ \\
6b & $0 / 6(0)$ & $0 / 6(0)$ & $0 / 6(0)$ \\
6c & $0 / 6(0)$ & $0 / 6(0)$ & $0 / 6(0)$ \\
Indomethacin & $3 / 6(1.4 \pm 0.2)^{\mathrm{bc}}$ & $5 / 6(1.9 \pm 0.2)^{\mathrm{b}, \mathrm{c}}$ & $6 / 6(2.1 \pm 0.2)^{\mathrm{b}, \mathrm{c}}$ \\
\hline
\end{tabular}

a Number of rats with lesions bigger than $0.5 \mathrm{~mm}$ in length per total number of rats.

b Mean ulcer lesion \pm SEM $(\mathrm{mm})(n=6)$ in parentheses.

c Significant difference at $p<0.05$ compared to the control.

\section{Acute toxicity}

$L D_{50}$ of compounds 6 a-c was found to be 145,155 and $165 \mathrm{mg} \mathrm{kg}^{-1}$ (i.p.), respectively, whereas, $L D_{50}$ of indomethacin was $50 \mathrm{mg} \mathrm{kg}^{-1}$ (i.p.). 


\section{CONCLUSIONS}

1-[(4-Chloro-2,2-dimethyl-2H-chromen-3-yl)methylene]thiosemicarbazide (6a), 1-[(4-chlorospiro- $2 H$-chromen $\left(2,1^{\prime}\right)$ cyclohexane-3-yl)methylene]thiosemicarbazide $(6 \mathbf{b})$ and 1-[(4-chloro-2-ethyl-2-methyl-2H-chromen-3-yl)methylene]thiosemicarbazide (6c) were found to be potent anti-inflammatory agents comparable to indomethacin. On the other hand, they show no ulcerative activity and higher $L D_{50}$ values than indomethacin. The thiosemicarbazide group at position 3 of the pyrane ring might be responsible for these activity.

\section{REFERENCES}

1. B. C. Sekhar, S. R. Ramadas and D. V. Ramana, $\beta$-Halovinylaldehydes as versatile reactive intermediates in the syntheses of condensed fused ring polycyclic heterocycles, Heterocycles 53 (2000) 941-977.

2. T. Aubert, M. Farnier, I. Meunier and R. Guilard, Synthesis of dipyrazolo[3,4- $\left.b: 4^{\prime}, 3^{\prime}-f\right]$ azepines and dithieno[2,3- $b: 3^{\prime}, 2^{\prime}$-f]azepines from 1-substituted 2,7-dichloro-4,5-dihydro-1H-azepine-3,6-dicarbaldehydes, J. Chem. Soc. Perkin Trans. I 1989, 2095-2098; DOI: 10.1039/p1989002095.

3. M. I. El-Deen, Use of 3-(2-formyl-1-chlorovinyl)coumarin in the synthesis of pyrazole and pyrimidine derivatives and salicylaldehyde azine, Chin. J. Chem. 17 (1999) 391-397.

4. M. I. El-Deen, K. H. Abd El-Fattah and I. A. El-Gohary, Synthesis and investigation of mass spectra of 3-[5'-(2'-substituent)thienyl]benzo[5,6]coumarines, Phosphorus Sulfur Silicon Relat. Elem. 178 (2003) 1463-1476; DOI: 10.1080/10426500307879.

5. K. Clarke, D. N. Gregory and R. M. Scrowston, Naphtho[1,2-b]thiophene. Part 1. Preparation and electrophilic substitution, J. Chem. Soc. Perkin Trans. I 1973, 2956-2960; DOI: 10.1039/ p19730002956.

6. M. I. Hegab and M. M. Abdulla, 4-Chloro-2,2-disubstituted chromene-3-carboxaldehyde: Synthesis of some fused polycyclic heterocycles as anti-inflammatory, analgesic, anticonvulsant, and antiparkinsonian agents, Arch. Pharm. Chem. Life Sci. 339 (2006) 41-47; DOI: 10.1002/ardp. 200500124

7. R. A. Pawar and A. P. Rajput, Studies on the Vilsmeier-Haack reaction. A versatile new synthesis of 4-chloro-2-phenylaminothiazole-5-carboxaldehyde and related fused heterocyclic compounds, Indian J. Chem. B 28 (1989) 866-867.

8. J. V. Dijk and J. M. A. Zwagemakers, Oxime ether derivatives, A new class of nonsteroidal anti-inflammatory compounds, J. Med. Chem. 20 (1977) 1199-1206; DOI: 10.1021/jm00219a018.

9. H. Chowdhury and V. S. Saxean, Synthesis and insect growth regulatory activity of alkoxy-substituted benzaldoxime ethers, J. Agr. Food Chem. 46 (1998) 731-736; DOI: 10.1021/jf970062h.

10. D. P. Jindal, R. Chattopadhaya, S. Guleria and R. Gupta, Synthesis and antineoplastic activity of 2-alkylaminoethyl derivatives of various steroidal oximes, Eur. J. Med. Chem. 38 (2003) 10251034; DOI: 10.1016/j.ljmech.2003.09.002.

11. C. Kirilmis, M. Koca, A. Cukurovali, M. Ahmedzade and C. Kazaz, Synthesis, reactivity and biological activity of novel bisbenzofuran-2-yl-methanone derivatives, Molecules 10 (2005) 13991408 .

12. F. A. G. El-Essawy, S. M. Yassin, I. A. El-Sakka, A. F. Khattab, I. Støfte, J. Møller and A. Senning, Cycloaddition with 2,2-dialkyl-3-thioxochroman-4-one S-sulfides including an unprecedented [3+5] cycloaddition, J. Org. Chem. 63 (1998) 9840-9845; DOI: 10.1021/jo9815347. 
13. H. J. Kabbe, Eine einfache Synthese von 4-Chromanonen, Synthesis 1978, 886-887; DOI: 10. 1055/s-1978-24924.

14. Alberola, B. Calvo, A. G. Ortega and R. Pedrosa, Reaction of 4-chlorocoumarin with organometallic reagents. Synthesis of trialkylbenzopyrans, 4-alkylcoumarins and o-hydroxyphenylprop-2-ynyl alcohols, J. Chem. Soc. Perkin Trans I 1992, 3075-3080; DOI: 10.1039/p19920003075.

15. T. Eszenyi, T. Tímár and P. Sebók, A novel synthesis of 4-halo-2H-chromenes, Tetrahedron Lett. 32 (1991) 827-828; DOI: 10.1016/S0040-4039(00)74898-2.

16. M. I. Hegab, A. M. Abdel-Fattah, N. M. Yousef, H. F. Nour, A. M. Mostafa and M. Ellithey, Synthesis, X-ray structure, and pharmacological activity of some 6,6-disubstituted chromeno[4,3-b]and chromeno[3,4-c]-quinolines, Arch. Pharm. Chem. Life Sci. 340 (2007) 396-403; DOI: 10.1002/ ardp. 200700089.

17. M. Hernández-Pérez, R. M. Rabanal, M. C. de la Torre and B. Rodriguez, Analgesic, anti-inflammatory, antipyretic and haematological effects of aethiopinone, and $o$-naphthoquinone diterpenoid from Salvia aethiopis roots and two hemisynthetic derivatives, Planta Med. 61 (1995) 505509; DOI: 10.1055 /s-2006-959358.

18. H. Ikuta, H. Shirota, S. Kobayashi, Y. Yamagishi, K. Yamada, I. Yamatsu and K. Katayama, Synthesis and anti-inflammatory activities of 3-(3,5-di-tert-butyl-4-hydroxybenzylidene)pyrrolidin-2-ones, J. Med. Chem. 30 (1987) 1995-1998; DOI: 10.1021/jm00394a011.

19. F. Sztaricskai, I. E. Takacs, F. Pusztai, G. Szabo and I. Csipo, Antiulcer effect of the $N-$ and $O-\beta$ D-glucopyranosides of 5-aminosalicylic acid, Arch. Pharm. 332 (1999) 321-326; DOI: 10.1002/ (SICI)1521-4184(19999)332:9<321::AID-ARDP321>3.3.CO;2-1.

20. J. Hlaváč, R. Buchtík, J. Slouka, P. Haradil and I. Wiedermannová, Synthesis of oxo analogs of Lamotrigine and related compounds, Arkivoc (2003) 22-28; ref. Chem. Abstr. 140 (2004) 199296p.

21. M. C. Pirung and S. V. Pansare, Trityl isothiocyanate support for solid-phase synthesis, J. Comb. Chem. 3 (2001) 90-96; DOI: 10.1021/cc000072m.

\section{$S A \check{Z} E T A K$}

\section{Sinteza i farmakološko djelovanje derivata kondenziranih 4-klor-2,2-dialkil kromen-3-karbaldehida}

MOHAMED IBRAHIM HEGAB, NABIL MAHMOUD YOUSEF, HANY FATHY NOUR, MOHEY ELLITHEY i MAHMOUD SOLIMAN ARBID

Novi hidrazono- 5a,b, tiosemikarbazono- 6a-c i oksimo kromeni 7a-c sintetizirani su iz odgovarajućeg $\beta$-klorkarbaldehida 3 i hidrazina, aromatskog hidrazina, tiosemikarbazida ili hidroksilamin hidroklorida, dok su eterski derivati 8a-h pripremljeni iz pripadajućih aldoksima 7a-c. Novi spojevi ispitani su na protuupalno i ulcerogeno djelovanje, a njihovo djelovanje uspoređeno je s djelovanjem indometacina.

Ključne riječi: $\beta$-klorkarbaldehid, hidrazoni, tiosemikarbazoni, oksim-eteri, protuupalno, ulcerogeno djelovanje

Photochemistry and Pharmacology Department, National Research Centre, Cairo, Egypt 